\title{
Débat avec les responsables scientifiques de l'axe 2
}

\section{Gino Gramaccia et François Pichault}

\section{OpenEdition}

Journals

Édition électronique

URL : http://journals.openedition.org/communicationorganisation/1995

DOI : 10.4000/communicationorganisation. 1995

ISSN : $1775-3546$

\section{Éditeur}

Presses universitaires de Bordeaux

\section{Édition imprimée}

Date de publication : 1 novembre 1997

ISSN : 1168-5549

\section{Référence électronique}

Gino Gramaccia et François Pichault, « Débat avec les responsables scientifiques de l'axe 2 », Communication et organisation [En ligne], 12 | 1997, mis en ligne le 26 mars 2012, consulté le 01 mai 2019. URL : http://journals.openedition.org/communicationorganisation/1995 ; DOI : 10.4000/ communicationorganisation.1995

Ce document a été généré automatiquement le 1 mai 2019.

(c) Presses universitaires de Bordeaux 


\title{
Débat avec les responsables scientifiques de l'axe 2
}

\author{
Gino Gramaccia et François Pichault
}

1 Gino Gramaccia: Je voudrais insister sur une question d'ordre général qui concerne le caractère introuvable des conditions méthodologiques qui devraient permettre d'établir une taxinomie argumentative. Il n'y a de dynamique argumentative que dans la langue. Dans sa typologie des contenus implicites, Catherine Kerbrat-Orecchioni, citant Ducrot, rappelle que des faits comme la présupposition ou encore l'orientation argumentative d'un énoncé ne peuvent être décrits que dans le cadre d'une problématique des actes de langage.

2 Encore faut-il en déterminer les marqueurs verbaux comme, par exemple, la coordination " mais ", ou non verbaux, comme les gestes allusifs susceptibles de suggérer une conclusion dans l'esprit de l'interlocuteur.

3 Cela dit, de la même manière qu'on n'a pas pu, à un moment donné, juguler l'hémorragie des actes de langage, ne peut-on stopper la prolifération des actes argumentatifs. Georges Vignaux, parlant de l'argumentation comme technique qui consiste à peser le pour et le contre, puis à en dégager une issue, dit qu'il s'agit d'une technique fondamentale et commune à toutes les entreprises de langage.

4 Votre projet d'établir des familles d'arguments présente un intérêt pratique évident, à la condition de raccrocher l'usage de certaines technique pré-argumentatives comme le "cadrage du réel ", à une théorie des actes du langage. Vous évoquez cette possibilité à propos de l'affirmation de l'autorité et de sa fonction dans le cadrage du réel.

5 N'y aurait-il pas précisément dans la langue et dans les usages de la langue des marqueurs sociaux, institutionnels, juridiques, susceptibles de fournir des critères de classification plus rigoureux?

6 Les linguistiques, les philosophes, les logiciens ont fait des travaux depuis longtemps, sur la rhétorique, l'argumentation. Ce qui m'intéresse c'est la place que peut avoir une théorie de l'argumentation spécifique au sein de la communication. Je pense qu'il manquerait quelque chose dans le champ de la communication si nous ne nous préoccupions pas de ces questions d'argumentation qui correspondent d'ailleurs à une demande sociale. 
7 Je suis sensible aux linguistes, mais je ne partage pas le point de vue de Ducrot qui dit « tout dans la langue est argumentation », je crois aux frontières et je crois qu'il y a autre chose dans la communication que l'argumentation.

8 Je prendrais un dialogue avec les linguistes qui serait de part et d'autre d'une frontière qui séparerait linguistique et sciences de la communication pour éviter toute confusion. La deuxième remarque est que je crois à une éthique de l'orateur. C'est un des points qui suscite le plus de difficultés, quand je dis que l'orateur à la co-resonsabilité de la relation qu'il établit. Il se place dans une position orgueilleuse, qui consiste à dire je vais vous convaincre. Cela implique, à mon avis, une éthique de l'orateur, de l'émetteur qui consiste partout où il le peut à diminuer son influence auprès du public. Parce que pour moi argumenter c'est laisser à l'auditoire la liberté la plus complète d'adhérer à l'opinion qu'on lui propose.

10 Ce qui est tout à fait paradoxal, car vous voulez convaincre et d'un autre côté vous créez une situation où vous laissez le choix, vous aménagez la liberté d'autrui. L'orateur est responsable de la liberté d'autrui et cette responsabilité est toujours parasitée : par son veston, son éventuelle prestance, ou la clarté de son rapport oratoire, son prestige, son autorité. Il me semble que l'éthique de l'orateur est l'éthique de la diminuation, du retrait. L'orateur doit se retirer par rapport à son opinion, car c'est son opinion qui compte.

11 À partir de là, on peut réfléchir à des critères. Sont-ils dans la langue ou dans la situation de communication? Les linguistes diraient qu'ils sont dans la langue avant d'être dans la situation de communication. Est-ce que nous ne devrons pas réfléchir à ce qui dans une éthique de la communication permettrait d'établir avec plus de précisions ces critères? Pour moi, il y a trois critères importants. Le premier c'est la liberté de l'auditoire. On est bien dans une problématique communicationnelle et non pas de langue, situation de communication où il faut à tout prix respecter la symétrie, ce principe démocratique essentiel, le respect de l'auditoire, le fait que l'auditoire puisse adhérer librement à la thèse qu'on lui propose.

Le deuxième point est un peu plus technique et concerne la conformité entre l'argument construit et l'opinion que l'on défend. Il doit y avoir une cohérence éthique et technique entre l'opinion que j'ai et le message que je mets en forme pour convaincre. Et la démagogie est justement la disjonction complète entre l'opinion que j'ai et le message que je construis. On voit bien d'ailleurs, qu'une partition a été établie, dans la publicité, entre des publicités qui assurent la cohérence entre l'opinion et le message et dans des publicités qui disjoignent complètement l'opinion et le message.

13 Le troisième point est qu'on n'argumente que du vraisemblable. C'est une partition difficile et toujours une question de frontière. On n'argumente que ce qui est discutable. C'est-à-dire qu'il faut sortir du domaine de l'argumentation ou il faut respecter d'autres règles, comme dans le domaine des sciences par exemple, ou bien religieux, du sentiment, de l'information. Tout n'est pas argumentation. Je plaiderais même pour la laïcité de l'argumentation, c'est-à-dire que tout ce que j'ai cité jusqu'à présent ne fait pas partie de l'espace public et l'argumentation au fond est un domaine laïque.

Dernier point : quels sont les critères qui détermineraient par exemple l'argumentation et la manipulation? Je crois qu'il y a là une réflexion tout à fait spécifique à conduire. Je suis persuadé que l'on peut établir des critères, ou une réflexion sur les critères, qui séparent argumentation et manipulation. Je suis en train de terminer un ouvrage sur la 
manipulation où j'essaye de montrer que les énoncés manipulatoires ne sont pas des arguments. J'essaye de proposer des critères pour distinguer ce qu'est un énoncé manipulatoire sur le mode affectif, sur le mode cognitif. Qu'est-ce qui distingue un énoncé manipulatoire d'un argument?

Et du coup, c'est une piste de réflexion très intéressante car on voit que les deux sont très liés; que l'argument et l'énoncé manipulatoire sont deux faces d'un même objet et qu'il n'y a pas entre les deux une ligne discontinue, mais qu'il y a entre les deux une zone intermédiaire, continue. Et celle-ci ouvre l'espace de notre responsabilité; bien évidemment s'il y avait des critères simples pour dire « ceci est un argument, ceci est un énoncé manipulatoire » nous n'aurions même pas besoin de réfléchir, nous appliquerions la norme. Or, justement ce n'est pas une norme mais un processus continu. On est dans l'argumentation et puis à un moment on bascule doucement vers quelque chose qui n'est plus de l'argumentation, qui commence à être de la manipulation.

16 Les cas extrêmes sont rares. Et nous évoluons toujours dans un univers qui convoque notre éthique et notre responsabilité individuelle. On est toujours renvoyé à soi-même pour construire ces critères.

17 Michèle Gabay: On peut lire votre présentation selon deux niveaux. Le niveau de l'individu et celui du politique avec son rôle social. Ce qui me paraittrait intéressant serait d'établir la manipulation dans l'interaction individuelle et la manipulation du politique. Comment délimitez-vous cette frontière entre les deux? Et pourriez-vous, par exemple, reprendre le risque de la démocratie que vous avez développé avec la rhétorique actuelle, la langue de bois par exemple.

18 François Pichault: Avant la réponse, je souhaiterais introduire un troisième niveau. Ce qui me frappe en observant la vie des organisations aujourd'hui c'est que, au fond, on est dans un contexte de panne de l'argumentation. Il y a eu une décennie, celle des années 80, qui a fortement misé sur l'argumentation, sur la mobilisation, sur la recherche de consensus des différents critères proposés, et fonctionnant sur le postulat d'une implication donnée dans l'entreprise sur la non-divergence des intérêts. Et puis là, brusquement c'est la panne de ce modèle. C'est l'implication qui passe du postulat au statut de problème. Aujourd'hui le gros problème dans la vie des organisations est d'obtenir l'implication des collaborateurs à qui on a plus rien à offrir en échange. À ce niveau intermédiaire, comment situer la question de l'argumentation.

19 Je crois que l'argumentation et la manipulation s'adressent toujours à des individus. Nous ne sommes pas autre chose que des individus. Simplement, la manipulation s'adresse à chaque individu comme s'il était l'élément d'une masse. Alors que l'argumentation s'adresse à l'individu en tant qu'individu. On voit bien que des techniques manipulatoires font disparaître en nous l'individu et flattent en nous la catégorie. Il y a dans la manipulation une évidente collusion avec ce qui a envahi le $\mathrm{XX}^{\mathrm{e}}$ siècle, la question de l'efficacité, de l'instrumentalisation. On instrumentalise en nous. Le grand auteur à redécouvrir c'est Pavlov, dont on a vraiment sous-estimé l'influence dans toute une série de courants. Pavlov s'adresse en nous à ce qui n'est pas le sujet, à ce qui n'est pas l'individu et c'est cela qui marche dans Pavlov. Donc, je ne verrais pas une forte opposition entre la dimension sociale et la dimension individuelle, mais comme cela a été souligné dans des études sur la propagande avant que celles-ci disparaissent comme inconvenantes. Je vous dis tout de suite que je vais essayer de faire revenir à la surface les études sur la propagande renouvelées aujourd'hui, car il me semble que notre espace public est envahi aujourd'hui de procédures manipulatoires que nous n'identifions pas comme telles; ce qui est d'ailleurs toujours le cas quand on est dans le bain de la propagande. On ne sait pas qu'on y est. C'est justement une de ses caractéristiques. 
Faisons tout, nous qui avons une responsabilité en communication, pour qu'on ne nous dise pas dans vingt ans : « vous étiez dans un régime manipulatoire, quasiment totalitaire du point de vue des méthodes, et vous ne nous l'avez pas dit ». Evidemment, la réflexion sur la manipulation rencontre un obstacle immédiat très fort. Il n'est pas drôle de dire aux gens et à soi-même : j'ai été manipulé. Le messager qui parle de la manipulation est toujours mal accueilli. Je veux bien m'en charger.

Panne de l'argumentation mais progrès de la manipulation à mon sens. Ce que vous dites sur l'implication du travailleur, je ne peux pas y répondre directement, car malgré ma place aujourd'hui dans cet atelier, je ne suis pas spécialiste de ce domaine; donc je l'éviterai avec une démarche inductive, en prenant encore une fois l'exemple de la démocratie grecque. Comme dit Emmanuel Terray, la grande invention de la démocratie grecque, c'est de découper dans l'espace social un espace particulier qui est l'espace du politique. La démocratie grecque constate que les hommes sont inégaux, socialement, intellectuellement, corporellement. Le mythe égalitaire démocratique n'est pas un aveuglement su les inégalités. Il les constate ces inégalités mais il se demande comment faire avec. Terray le dit très bien, c'est un exercice de découpage. On découpe dans l'espace social un petit carré, espace du politique et on décrète que dans ce carré tout le monde est égal. Quelle que soit l'inégalité quand on est à l'extérieur de ce carré. Ce qui pose un problème insurmontable à la démocratie : une voix en vaut-elle vraiment une autre? Oui et non. C'est évidemment une question très délicate. Je me permets cette comparaison avec l'exemple que vous proposez, celui du découpage de l'entreprise, d'un espace à l'intérieur duquel on décrète que tout le monde est égal, peut tout dire, mais en dehors de cet espace les hiérarchies et les inégalités reviennent. Alors, le problème de la démocratie est: comment gérer les inégalités extérieures au politique avec l'égalité intérieure au politique? Nous ne savons pas bien faire, étant entendu qu'il faut diminuer les inégalités mais tant que cela n'est pas fait, comment garantir un bon fonctionnement du politique qui reste inégalitaire?

\section{AUTEURS}

GINO GRAMACCIA

Université Bordeaux 1, IUT A - Dpt. Génie électrique

FRANÇOIS PICHAULT

Université de Liège, LENTIC, Belgique 